\title{
Oblique shock interaction with a cylindrical density interface
}

 \\ ${ }^{I}$ Department of Mechanical Engineering, The University of New Mexico, \\ USA \\ ${ }^{2}$ Department of Engineering, The University of Texas at Brownsville, USA
}

\begin{abstract}
A cylindrical, initially diffuse density interface is formed by injecting a laminar jet of heavy gas into the test section of a shock tube. The injected gas is mixed with a fluorescent gaseous tracer, small liquid droplets, or smoke particles. The shock tube is tilted with respect to the horizontal. Thus the axis of the gravitystabilized heavy gas jet is at an oblique angle with the plane of the arriving shock front. The flow structure forming after the oblique shock wave interaction with the column of heavy gas is revealed by visualization in multiple planes. We observe the formation of the well-known counter-rotating vortex columns (same as caused by normal shock waves). However, along with them, periodic co-rotating vortices form in the vertical plane in the flow downstream of the oblique shock. The size of these vortices varies both with the Mach number and with the initial angle between the column and the shock front.

Keywords: shock waves, Kelvin-Helmholtz, Richtmyer-Meshkov, PLIF, vorticity deposition, oblique shock, wavelength, turning angle.
\end{abstract}

\section{Introduction}

Richtmyer-Meshkov instability (RMI) occurs when an interface between two fluids of different densities is impulsively accelerated. This acceleration can be the result of an impulsive body force, or a passing shock wave [1]. RMI develops on the interface between a light fluid and a heavy fluid, regardless of the respective direction of the density gradient and the acceleration. This instability is physically relevant for many natural and engineering phenomena, including evolution of 
supernova remnants, deflagration to detonation transition, inertial confinement fusion (ICF). RMI also plays an important role in mixing during combustion (for a summary, refer to Refs. [2,3] and citations therein). RMI usually develops in combination with several other hydrodynamic instabilities, one of which is the well-known Kelvin-Helmholtz instability (KHI). KHI occurs when there is a significant velocity difference on the interface between two fluids. Shear created at the interface by this velocity difference induces vorticity which eventually leads to the formation of "cat's eye" vortices [4]. KHI is present in the cloud bands of giant planets and has been observed in targets accelerated by laser ablation [5].

The recent experiments conducted at the shock tube facility at the University of New Mexico have been focused on oblique shock interactions with a column of heavy gas (sulfur hexafluoride $\mathrm{SF}_{6}$ ) surrounded by air. The oblique shock-accelerated gas columns reveal the expected evolution of Richtmyer-Meshkov instabilities, with many features similar to earlier studies with quasi-two-dimensional initial conditions. The oblique case also possesses small-scale three-dimensional (3D) features, physically and morphologically identical to Kelvin-Helmholtz vortices, that develop on the edges of the column. These instabilities present themselves as periodic waves that grow with time and evolve into vortices that cascade down the entire length of the gas column. This paper focuses on these small-scale KelvinHelmholtz instabilities, including a description of the experimental setup, data for $20^{\circ}$ and $30^{\circ}$ oblique shock angles, and a detailed description of the periodic behavior, including Mach number dependence.

\section{Experimental arrangement and diagnostics}

The University of New Mexico Shock Tube Facility is primarily dedicated to the study of RMI. It has been used in several studies of shock wave interactions with a multiphase or heavy gas column [6,7]. The shock tube itself can operate in a horizontal position or can be inclined to any angle $\theta$, up to $45^{\circ}$ above horizontal. Fig. 1 shows the shock tube inclined to $30^{\circ}$. Under normal operation, the driver section is pressurized with helium to a predetermined pressure (depending on the desired Mach number). A thin polyester diaphragm separating the driver and driven sections is then punctured, sending a planar normal shock down the length of the tube. Two pressure transducers, located approximately $2.6 \mathrm{~m}$ apart on the top of the driven section, record the pressure pulse from the shock wave as it passes. This information is displayed, stored, and is used to trigger the diagnostics and verify the velocity of the shock. The inclined angle of the shock tube, coupled with the planar normal shock impacting the initial conditions (oriented vertically and stabilized by gravity), results in an oblique interaction between the shock and the density interface.

In the experiments described here, initial conditions (ICs) in the test section of the shock tube are formed by vertical injection of sulfur-hexafluoride $\left(\mathrm{SF}_{6}\right)$ infused with about $1 \%$ acetone gas. The governing parameters of each experiment are the Mach number and the Atwood number. The Mach number here is $M=U / a$, where $U$ is the shock front speed as it propagates through the driven section and 




Figure 1: Shock tube inclined to $30^{\circ}$. The driver and driven sections are separated by a polyester diaphragm. Pressure transducers are located on top of the driven section. Flow direction is from left to right.

$a$ is the speed of sound in air. The Atwood number is $A=\left(\rho_{2}-\rho_{1}\right) /\left(\rho_{2}+\rho_{1}\right)$, where $\rho_{2}$ and $\rho_{1}$ are the densities of the heavy and light gas, respectively. For $\mathrm{SF}_{6}$ and air, $A=0.67$.

The shock-accelerated column of heavy gas is illuminated by a New Wave Research Gemini 200 UV laser. Planar Laser-Induced Fluorescence (PLIF) images of the column are collected using a four-megapixel Apogee Alta U-42 astronomy camera, with a nominal quantum efficiency of $90 \%$.

The Mach number for each experiment varies by no more than $0.5 \%$ from the nominal value, and up to 20 images per Mach number and $\theta$ have been used to produce the results presented in the next section, as each experimental run produced one dynamic image at a prescribed timing after shock acceleration.

\section{Results and discussion}

Misalignment of the pressure $p$ and density $\rho$ gradients in the column of heavy gas accelerated by an oblique shock results in three-dimensional vorticity deposition (Fig. 2). The baroclinic vorticity deposited by the shock interaction is proportional to the cross-product $\nabla \rho \times \nabla p$. While in the plane of the cross-section of the column (Fig. 2a, top right), this leads to formation of a counter-rotating vortex pair, in the vertical plane (Fig. 2a, bottom right), vortex sheets form on the upstream and downstream sides of the column. 
As the result, shear layers form on the density interfaces in the vertical plane (Fig. 2b), leading to velocity differences between the lighter air and the heavier column material, and to formation of KHI. For example, the air is traveling down the upstream side of the column, resulting in small-amplitude perturbations evolving into vortices. The kinetic energy of the air moving down the edge of the column fuels the vortex growth via shear.

(a)

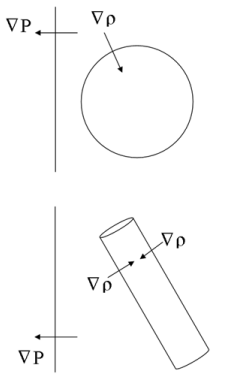

(b)

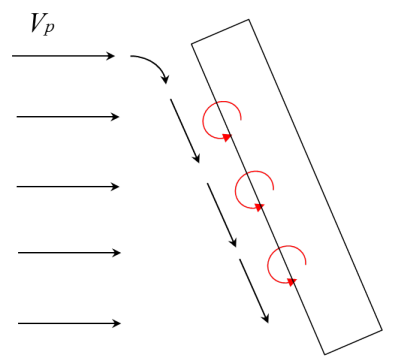

Figure 2: Sketch of three-dimensional vorticity deposition showing (a) pressure and density gradients [8] and (b) air moving down the upstream edge of the column.

High repeatability of the initial conditions allows to reconstruct the time history of the flow based on analysis of the experimental images acquired at different times after shock arrival for each prescribed $M$ and $\theta$. The quantitative parameter of interest here is the wavelength $\lambda$ of KHI. Wavelengths are measured as the linear distance between the centers of consecutive vortices using ImageJ software [9]. The measurement area is $1.0 \mathrm{~cm} \times 1.5 \mathrm{~cm}$ (Fig. 3) both for the $\theta=20^{\circ}$ and $\theta=30^{\circ}$ cases, allowing to use a sequence of five consecutive vortices to produce a total of four measurements per image. Measurements were then averaged for each image and sorted according to $\theta$ and $M$. Temporal evolution of the flow was presented in terms of non-dimensional time parameter $\tau$, derived from the linear growth theory of RMI [10]:

$$
\tau=k A \Delta V\left(t-t_{0}\right)
$$

Here $k=2 \pi / d$ is the perturbation wavenumber, where $d$ is the diameter of the initial conditions (and the dominant length scale), $\Delta V$ is the Mach numberdependent piston velocity, and the expression $\left(t-t_{0}\right)$ is the time after shock arrival at the center of the heavy gas column.

Figure 4 shows a sequence of six vertical plane images captured at a $30^{\circ}$ inclination angle at Mach number $M=2.00$. KHI first presents itself as small perturbations at the top of the column and moves vertically down the upstream edge $(t=90 \mu \mathrm{s}$ after shock impact). The instabilities quickly evolve into 
(a)

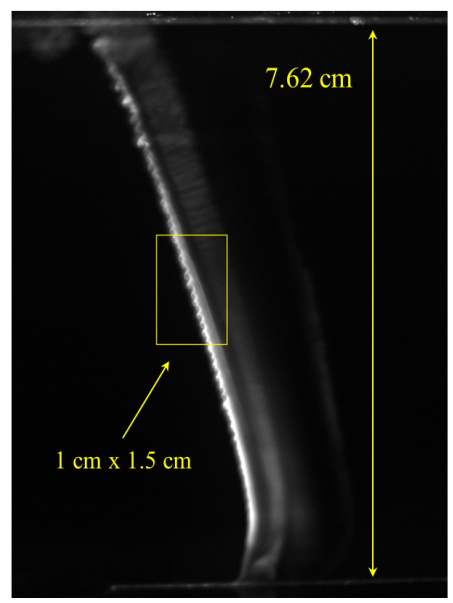

(b)

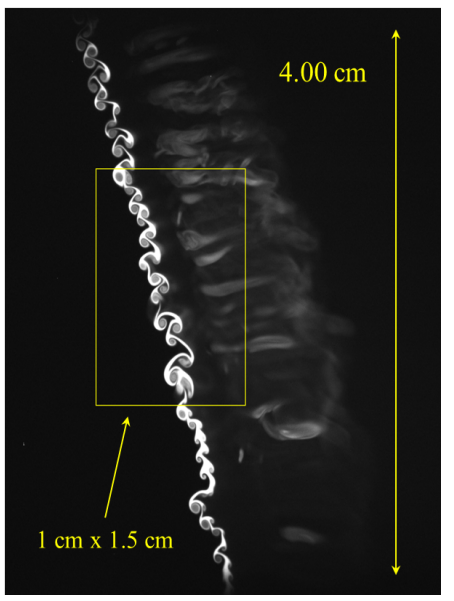

Figure 3: Areas of interest where measurements of KHI were taken for (a) $30^{\circ}$ and (b) $20^{\circ}$ inclination angles. The full cross-sectional extent of the test section of the shock tube is $7.62 \mathrm{~cm}$, images at $20^{\circ}$ were taken at a higher resolution, but the area of interest has the same physical dimensions.

"cat's eye" vortices, indicative of KHI $(t=99,100 \mu \mathrm{s})$. At later times, same-sign vortices begin to merge ( $t=112 \mu \mathrm{s})$. The resulting larger-scale vortices start to $\operatorname{mix}(t=124 \mu \mathrm{s})$, resulting in apparent transition to turbulence $(t=180 \mu \mathrm{s})$.

Figure 5 shows a comparison of KHI vortices observed at several Mach numbers at a $30^{\circ}$ inclination angle. As the Mach number increases, the respective wavelength decreases. This behavior is expected since the dominant length scale amplified by the $\mathrm{KHI}$ is the size of the cross-section of the shock-compressed $\mathrm{SF}_{6}$ column. At higher Mach numbers, there is greater compression and accordingly the dominant post-shock length scale is reduced [8].

Similar trends can be observed in KHI images acquired at a $20^{\circ}$ inclination angle, with one notable difference. For a Mach $=1.13$ shock wave, the waves that formed on both sides of the high-density region (Fig. 6), soon evolving to produce staggered counter-rotating vortex pairs. This behavior precipitated mixing and turbulent transition for this case. Images at later times reveal turbulent transition occurring well before that at $30^{\circ}$ inclination for the same Mach number.

Measurements of wavelength $\lambda$ are presented in Fig. 7. Here wavelength is normalized by the nominal (pre-shock) diameter of the initial conditions. Horizontal error bars correspond to standard deviation of Mach number while the vertical error bars are the standard deviation of $\lambda$. Power-law fits are superimposed with each data set (Fig. $7 \mathrm{a}, \theta=30^{\circ}$, and Fig. $7 \mathrm{~b}, \theta=20^{\circ}$ ) to serve as guides for the eye.

For an inclination angle $\theta=30^{\circ}$, wavelengths ranged from $1.60 \mathrm{~mm}$ for Mach 1.13 to $0.97 \mathrm{~mm}$ for Mach 2.00. The average standard deviation of $\lambda$ for $30^{\circ}$ and $20^{\circ}$ inclination angles is $101.2 \mu \mathrm{m}$ and $43.6 \mu \mathrm{m}$ respectively. Measurements 

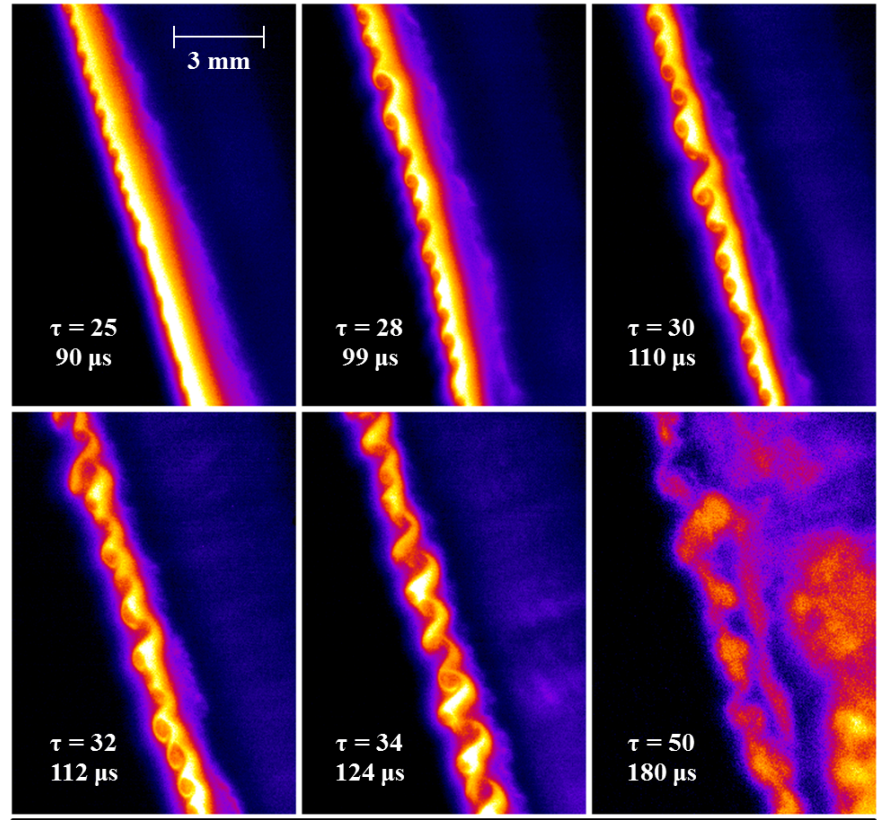

Air

Pure $\mathrm{SF}_{6}$

Figure 4: Temporal evolution of KHI for $M=2.00, \theta=30^{\circ}$. Each image is labeled with actual time after shock acceleration and dimensionless time $\tau$ (Eq. 1).

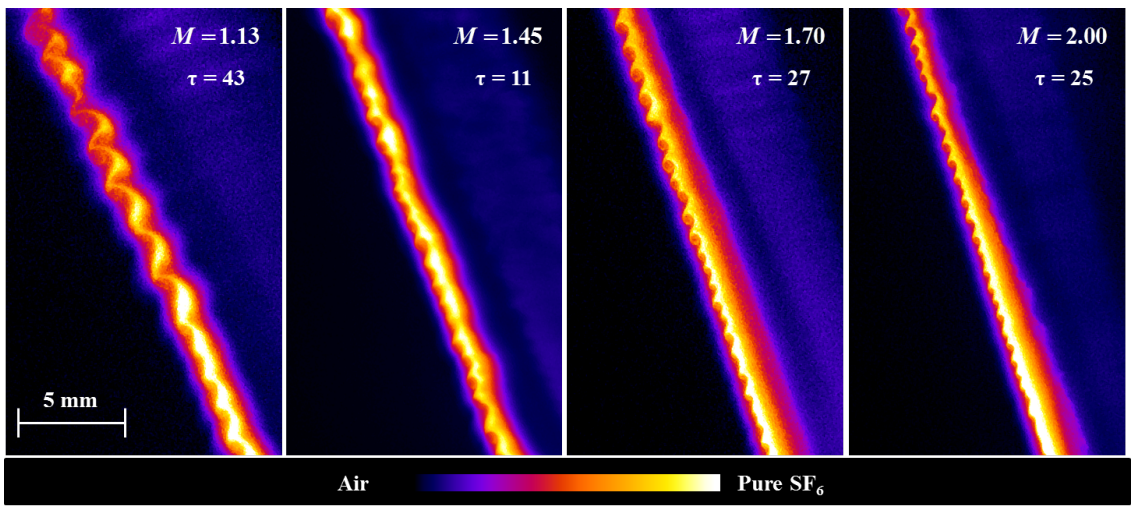

Figure 5: Comparison of KHI vortices at different Mach numbers (as labeled) for $30^{\circ}$ inclined shock tube. Mach number increases from left to right. 




Figure 6: Comparison of KHI vortices at different Mach numbers (as labeled) for $30^{\circ}$ inclined shock tube. At $M=1.13$, vortices form on both sides of the high density region.

taken at $20^{\circ}$ show that the wavelengths are significantly smaller than those at $30^{\circ}$ inclination, varying by as much as $49 \%$. For Mach $=1.13, \lambda=1.10 \mathrm{~mm}$, and for Mach $=2.00, \lambda=0.49 \mathrm{~mm}$.

(a)

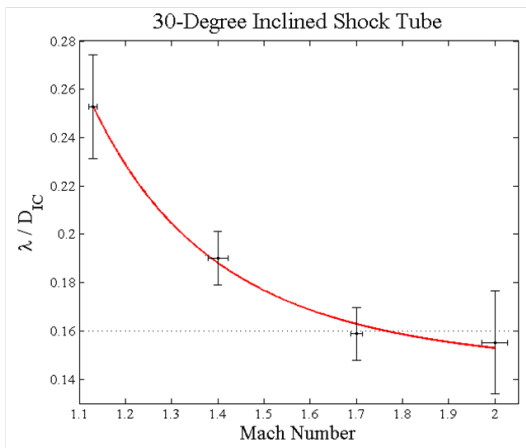

(b)

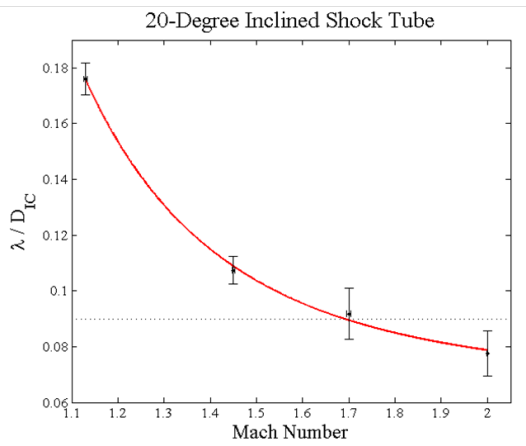

Figure 7: KHI wavelength $\lambda$ vs. Mach number $M$ for (a) $30^{\circ}$ and (b) $20^{\circ}$ inclination. The data suggest that $\lambda$ decreases as inclination angle $\theta \rightarrow 0$. Power-law fits are used as a visual aid.

While existing theory [8] explains the Mach number dependence for each $\theta$, explanation of the smaller scale of vortices for $\theta=20^{\circ}$ will require additional consideration. In the limit case of a planar normal shock $(\theta=0)$, there would be no macroscopic baroclinic vorticity deposition in the vertical plane, and thus no shear layers. It is possible that the length scale amplified by the KHI is not the compressed size of the gas column $d_{c}$, but rather this length scale projected on the 
direction along the column where KHI evolves, $d_{c} \tan \theta_{c}$, where $\theta_{c}$ is the tilt angle of the shock-accelerated column.

\section{Conclusions}

We have conducted an experimental study of the formation of Kelvin-Helmholtz secondary instability in the flow produced by oblique planar shock interaction with an initially cylindrical density interface. While some of the results are well explained by existing theory, additional studies will be conducted to clarify the nature of the observed relationship between the dominant KHI wavelength and the tilt angle of the gas column with respect to the shock front.

\section{Acknowledgement}

This research is supported by the US National Nuclear Security Administration (NNSA) grant DE-NA-0002220.

\section{References}

[1] C.E. Niederhaus. Experiments on the Richtmyer-Meshkov instability of incompressible fluids. $\mathrm{PhD}$ Dissertation, Aerospace and Mechanical Engineering, University of Arizona, 2000.

[2] M. Brouillette. The Richtmyer-Meshkov instability. Annual Review of Fluid Mechanics, 34(1):445-468, 2002.

[3] S. Kumar and P. Vorobieff. Experimental studies of Richtmyer-Meshkov instability. Recent Research Developments in Fluid Dynamics, 5:33-55, 2004.

[4] D. Ryu, T.W. Jones, and A. Frank. The magnetohydrodynamic KelvinHelmholtz instability: a three-dimensional study of nonlinear evolution. The Astrophysical Journal, 545(1):475, 2000.

[5] M. Emery, J. Gardner, and J. Boris. Rayleigh-Taylor and Kelvin-Helmholtz instabilities in targets accelerated by laser ablation. Phys. Rev. Lett., 48:677680, Mar 1982.

[6] T. Bernard, C.R. Truman, P. Vorobieff, C. Corbin, P. Wayne, G. Kuehner, M. Anderson, and S. Kumar. Observation of the development of secondary features in a Richtmyer-Meshkov instability driven flow. Journal of Fluid Engineering, 137(1):011206, 2015.

[7] P. Vorobieff, M. Anderson, J. Conroy, R. White, C.R. Truman, and S. Kumar. Analogues of Rayleigh-Taylor and Richtmyer-Meshkov instabilities in flows with nonuniform particle and droplet seeding. Computational Methods in Multiphase Flow VI, eds. AA Mammoli, CA Brebbia, WIT Press, Southampton, UK, pages 17-28, 2011.

[8] P. Wayne, D. Olmstead, P. Vorobieff, S. Kumar, and C.R. Truman. Experimental study of shock accelerated inclined heavy gas cylinder. Under review, Physics of Fluids, 2015. 
[9] T. Fereira and W. Rasband. ImageJ user guide. http://imagej.nih.gov/ij/ docs/guide/user-guide.pdf, 2012.

[10] R.D. Richtmyer. Taylor instability in shock acceleration of compressible fluids. Technical Report LA-1914, Scientific Laboratory of the University of California, Los Alamos, New Mexico, July 1954. 